\title{
Agente de liaison avec les personnes atteintes d'arthrite : un rôle important dans la prestation de soins à des patients des collectivités des Premières Nations
}

\author{
Valerie Umaefulam, Ph. D. (1); Adalberto Loyola-Sanchez, M.D. (1); Valene Bear Chief, inf. aux. aut. (2); \\ Ana Rame, Ph. D. (1); Louise Crane, B.A. (3); Tessa Kleissen, B. Sc. inf. (1); Lynden Crowshoe, M.D. (1); \\ Tyler White (2); Diane Lacaille, M.D. (4,5); Cheryl Barnabe, M.D. (1,5)
}

Diffuser cet article sur Twitter

\section{Résumé}

Introduction. L'arthrite est l'une des principales causes d'invalidité dans les collectivités des Premières Nations et elle s'accompagne souvent d'autres maladies chroniques. Les modèles de soins actuels donnent la priorité à l'accessibilité aux soins de traitement spécialisés alors que les approches centrées sur le patient ciblent des objectifs plus larges en matière de santé.

Méthodologie. Un modèle de soins fondé sur une facilitatrice des soins, appelée «agente de liaison avec les personnes atteintes d'arthrite», a été conçu en collaboration avec la collectivité pour créer des plans de soins axés sur le patient et adaptés à la culture. Au bout d'un an d'intervention, nous rendons compte de la faisabilité et de l'acceptabilité de ce modèle de soins du point de vue des patients et des fournisseurs de soins de santé.

Résultats. L'agente de liaison avec les personnes atteintes d'arthrite a fait le pont entre les cliniciens et les patients et a favorisé la continuité des soins, de manière à aider les patients à recevoir des soins coordonnés au sein de la collectivité.

Mots-clés : arthrite, maladie chronique, orientation des patients, Premières Nations, services de santé aux Autochtones, évaluation des résultats et des processus des soins de santé, étude qualitative

\section{Introduction}

On note une augmentation de la prévalence $^{1,2}$, de la gravitéz,4 et du sous-traitement de l'arthrite ${ }^{5}$ au sein des populations des Premières Nations. Les inégalités dans les déterminants de la santé découlant de la colonisation $^{6}$ ont une incidence sur l'état de santé7. Les fournisseurs de soins de santé nient l'autonomisation (empowerment) des patients, que ce soit de manière consciente (en ne tenant pas compte de leurs valeurs culturelles) ou inconsciente (en ne leur fournissant pas de soins adaptés à leur culture $)^{8}$. Cela a vraisemblablement une incidence sur la continuité des soins et mine les efforts visant à soutenir une gestion thérapeutique efficace et à favoriser le bien-être? .

Pour améliorer l'état de santé des membres des Premières Nations atteints d'arthrite ${ }^{10}$, il faut mettre en place des moyens novateurs de prestation de soins pertinents,

\section{Points saillants}

- Les modèles de soins qui abordent la santé d'une manière adaptée à la culture et qui s'attaquent également aux nombreux obstacles à la prestation de soins comblent plus efficacement les lacunes en matière de soins et optimisent les résultats de santé des membres des Premières Nations souffrant d'arthrite et de comorbidités.

- Le modèle de soins fondé sur la présence d'une agente de liaison avec les personnes atteintes d'arthrite a été créé à partir des besoins de prise en charge des cas définis par les membres des collectivités autochtones afin d'assurer que la facilitation de la prestation de soins soit incluse dans le modèle.

- Le modèle a couvert un éventail de rôles, allant des aspects médicaux au soutien personnel, et a suivi une approche adaptée à la culture, axée sur le patient et dictée par les besoins des patients.

aptes à surmonter les barrières sociales, géographiques et économiques. Conformément aux principes d'autonomisation et d'autodétermination des collectivités, nous devons donner la priorité aux projets

Rattachement des auteurs :

1. École de médecine Cumming, Université de Calgary, Calgary (Alberta), Canada

2. Siksika Health Services, Siksika (Alberta), Canada

3. Représentante indépendante des patients

4. Faculté de médecine, Université de la Colombie-Britannique, Vancouver (Colombie-Britannique), Canada

5. Arthritis Research Canada, Richmond (Colombie-Britannique), Canada

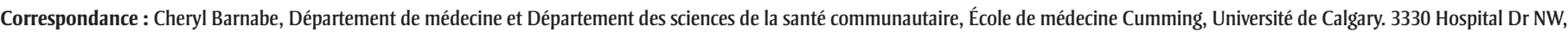
Calgary (Alberta) T2N 4N1; tél. : 403-220-3380; courriel : ccbarnab@ucalgary.ca 
dirigés par des Autochtones ${ }^{11}$ et aux projets qui valorisent les fournisseurs de soins de santé autochtones au sein du personnel ${ }^{12}$.

Les modèles de soins fondés sur la prise en charge des cas et ceux fondés sur l'orientation des patients au sein des systèmes de soins de santé, qui sont étroitement liés, constituent des approches efficaces dans la prise en charge des maladies chroniques ${ }^{13-16}$, mais les avantages de ces modèles sont mitigés en ce qui concerne la prise en charge de l'arthrite ${ }^{17-19}$. En outre, ils n’ont pas été mis en œuvre ni évalués dans le contexte des soins prodigués aux patients autochtones atteints d'arthrite. Ces modèles, qui visent à ce que les soins spécialisés soient moins axés sur chaque maladie séparément et qu'ils soient compatibles avec l'adoption de pratiques culturelles, pourraient constituer selon nous un moyen de soigner les personnes atteintes d'arthrite dans les collectivités autochtones.

Dans cet article, nous décrivons l'élaboration et la mise en œuvre d'un modèle de soins reposant sur une agente de liaison avec les personnes atteintes d'arthrite dans une collectivité des Premières Nations et nous présentons une évaluation de l'accessibilité de ce modèle.

\section{Méthodologie}

\section{Contexte}

Siksika Health Services est une organisation qui offre une gamme complète de soins de santé primaires autodéterminés. L'organisation offre une clinique communautaire spécialisée en rhumatologie depuis 2010. L'objectif de la clinique est de faciliter l'accès à des services d'évaluation et de traitements offerts par des spécialistes dans le cadre de soins primaires ${ }^{20}$. L'équipe de recherche a comparé la qualité des traitements donnés par cette clinique aux indicateurs de rendement approuvés à l'échelle nationale $e^{21}$. Nous avons également cherché à connaître le point de vue des utilisateurs des services sur les lacunes qui subsistent dans le traitement de l'arthrite ${ }^{22}$. Les points de vue recueillis ont confirmé la nécessité d'enrichir le modèle de soins en fournissant des services qui améliorent les fonctions physiques et mentales, en améliorant la communication, la continuité des soins et la sensibilisation de la population et en renforçant les liens entre les pairs et le soutien des pairs ${ }^{22}$.

\section{Co-création de l'intervention avec les membres de la collectivité}

L'équipe de recherche a proposé une intervention reposant sur un modèle de gestion de cas axé sur l'évaluation des besoins et l'élaboration de plans de traitement et de soins, en connectant les clients aux divers services, à un système de suivi et de soutien pour la défense de leurs intérêts ${ }^{23}$. Nous avons mis sur pied un groupe consultatif de recherche comprenant des membres de la collectivité et des Aînés souffrant d'arthrite, des représentants des Siksika Health Services et l'ancien représentant des patients autochtones de l'Alliance canadienne des arthritiques. Le chercheur principal de l'équipe de recherche est métis.

$\mathrm{Au}$ lieu de "gestionnaire de cas», la communauté a choisi le terme "agent(e) de liaison avec les personnes atteintes d'arthrite». Le groupe consultatif de recherche a décrit les responsabilités de cet agent de liaison, les stratégies de recrutement des patients et les différents volets de l'évaluation de la recherche. Nous avons embauché comme agente de liaison avec les personnes atteintes d'arthrite une membre de la collectivité ayant de l'expérience en soins infirmiers et nous l'avons formée afin qu'elle réponde aux besoins en matière de soins de l'arthrite et de compétences d'autogestion de l'arthrite. En outre, elle a suivi un cours d'une semaine sur les techniques d'entrevue motivationnelle et la mise en œuvre de plans d'action.

\section{Mise en ceuvre}

L'agente de liaison avec les personnes atteintes d'arthrite a fourni un soutien médical et personnel pendant un an, soutien qui impliquait de communiquer régulièrement avec les participants et leur équipe soignante (en personne, par téléphone ou par messagerie texte). Elle a également fourni du soutien à la clinique mensuelle spécialisée dans l'arthrite, en administrant les injections prescrites et en animant des activités de groupe pour les patients et leurs familles.

\section{Participants}

Nous avons recruté pour l'intervention des patients de la Nation Siksika qui souffraient d'arthrite inflammatoire ou d'arthrose et qui fréquentaient la clinique communautaire de rhumatologie du Siksika Health and Wellness Centre. Les patients devaient avoir 19 ans ou plus et, outre leur(s) affection(s) arthritique(s), devaient souffrir d'au moins l'une des maladies suivantes : diabète, obésité, hypertension, maladie cardiovasculaire ou bronchopneumopathie chronique obstructive.

\section{Évaluation et analyse de l'accessibilité}

Nous avons consigné le nombre d'interactions entre l'agente de liaison avec les personnes atteintes d'arthrite et les participants ainsi que les raisons de ces interactions. Nous avons utilisé des méthodes qualitatives pour explorer, du point de vue de chaque participant et des fournisseurs de soins de santé dans la collectivité, la manière dont l'agente de liaison a assuré les soins auprès des personnes atteintes d'arthrite. Nous avons enregistré les entrevues semi-structurées puis deux chercheurs les ont transcrites et codées de façon indépendante à l'aide du logiciel Dedoose (SocioCultural Research Consultants, LLC, Los Angeles, Californie). Nous avons comparé les codes pour vérifier la cohérence et la fiabilité interévaluateurs. Nous avons réalisé une analyse thématique et en avons interpréré les résultats à la lumière des principes des soins axés sur le patient et des concepts de "soins adaptés à la culture des peuples autochtones du Canada $»^{24}$. Comme l'agente de liaison avec les personnes atteintes d'arthrite était une femme, on a utilisé le pronom «elle» pour la désigner dans les citations tirées de des entrevues.

\section{Éthique}

L'étude a été approuvée par la direction des Siksika Health Services et par le Conjoint Health Research Ethics Board de l'Université de Calgary (REB 15-1961). Les rôles et responsabilités de l'équipe de recherche à l'égard de la collectivité ont été consignés dans un protocole d'entente. Tous les participants ont fourni leur consentement par écrit. La recherche a été réalisée conformément aux principes de propriété, de contrôle, d'accès et de possession, dits principes de PCAP, en matière de recherche des Premières Nations ${ }^{25}$, ainsi qu'à la Déclaration d'Helsinki de 1964 et à ses modifications subséquentes.

\section{Résultats}

\section{Participants}

Entre mai 2017 et décembre 2018, 23 patients se sont inscrits à l'étude. Les participants étaient principalement des femmes (80\%) 
dont l'âge moyen était de 59 ans (écarttype : 12 ans). La polyarthrite rhumatoïde $(\mathrm{n}=13)$ et la polyarthrite psoriasique $(\mathrm{n}=5)$ étaient les types d'arthrite les plus fréquents, avec comme comorbidités les plus fréquentes le diabète $(n=9)$ et l'hypertension $(n=6)$. Dix-sept patients ont participé à l'ensemble de l'intervention (deux se sont retirés, un est décédé et trois ont été perdus de vue).

\section{Accessibilité}

La fréquence et les types d'interaction entre l'agente de liaison avec les personnes atteintes d'arthrite, les participants et l'équipe soignante sont présentés dans le tableau 1.

Les témoignages des 14 participants, des 10 fournisseurs de soins et de l'agente de liaison avec les personnes atteintes d'arthrite ont montré que le modèle de soins améliorait l'accessibilité et était accepté et considéré comme adapté à la culture.

Les participants se sont dits satisfaits de la souplesse des communications et des interactions avec l'agente de liaison avec les personnes atteintes d'arthrite, qui s'est adaptée à leurs besoins et à leur situation. Parmi les exemples d'adaptation, citons les lieux de rencontre pour les injections et pour les suivis (p. ex. visites à domicile, rencontres pour boire un café), les méthodes de communication utilisées (p. ex. message texte, téléphone), l'étendue et la fréquence des communications ainsi que les styles de communication (p. ex. formel et informel).

La confiance envers l'agente de liaison avec les personnes atteintes d'arthrite, qui est le fruit d'interactions fondées sur le respect, le soutien, la compassion et la responsabilité, a aidé les participants à s'engager envers leur traitement. L'agente de liaison avec les personnes atteintes d'arthrite a communiqué avec divers fournisseurs de soins de santé appartenant au cercle de soins du patient afin de défendre les intérêts de ce dernier ou d'obtenir des ressources supplémentaires, notamment en organisant des consultations de fournisseurs de services paramédicaux, en conduisant le patient à des rendez-vous, en l'aidant à obtenir des médicaments et en étant présente lors de rendez-vous médicaux.

Enfin, au moyen de plans d'action, de rappels et d'activités de facilitation, l'agente de liaison avec les personnes atteintes d'arthrite a contribué à l'engagement du patient envers ses plans de soins, par exemple en facilitant l'accès aux ressources ou l'atteinte des objectifs fixés.

Voici divers thèmes portant sur l'accessibilité du modèle de soins fondé sur une agente de liaison avec les personnes atteintes d'arthrite illustrés par des citations (traduites) de participants-patients et de fournisseurs de soins.

\section{Faire participer les patients en fonction de leurs besoins et préférences}

[...] elle vérifie si tout va bien. Vous savez, elle veille à ce que nous ne manquions de rien. Si nous avons besoin de quelque chose ou si quelque chose se passe, nous n'avons qu'à l'appeler. (Participant 23)

Elle est facile à côtoyer, et je suis heureuse qu'elle ait fait preuve d'ouverture et de souplesse par rapport à mon emploi du temps. Par exemple, si nous avions rendez-vous à 14 heures

\section{TABLEAU 1}

Aperçu des interactions de l'agente de liaison avec les personnes atteintes d'arthrite dans la prestation des soins

\begin{tabular}{|c|c|}
\hline Type d'interaction & Nombre moyen (écart-type) \\
\hline En personne & 8 (5) heures par participant \\
\hline Messages textes & $\begin{array}{l}70 \text { (58) messages à chaque participant } \\
\text { (fourchette : } 9 \text { à 194) }\end{array}$ \\
\hline Visites à domicile & 112 \\
\hline Évaluations de santé & 74 \\
\hline Brefs plans d'action & De 2 à 5 objectifs par patient \\
\hline $\begin{array}{l}\text { L'agente de liaison avec les personnes atteintes } \\
\text { d'arthrite a accompagné le patient à son } \\
\text { rendez-vous ou a communiqué avec le fournisseur } \\
\text { de soins de santé pour défendre ses intérêts }\end{array}$ & $\begin{array}{l}168 \text { rendez-vous au total (soit entre } 1 \text { et } 7 \text { par } \\
\text { participant) portant sur les soins primaires ( } 8 \text { ), les } \\
\text { soins paramédicaux ( } 27) \text {, les soins à domicile ( } 23) \text {, le } \\
\text { transport médical (18), une enquête (2), un } \\
\text { rendez-vous avec un spécialiste (90) }\end{array}$ \\
\hline
\end{tabular}

et que j'avais un atelier ailleurs au même moment, elle se montrait tout à fait compréhensive. (Participant 01)

Elle répond immédiatement, et si ce n’est pas immédiatement, elle répond dans l'heure qui suit et même plus rapidement encore par message texte. (Participant 18)

Elle est un point de contact assez important pour les patients. D'après ce que les gens me disent, ils savent comment la joindre, et ils peuvent rapidement obtenir des réponses ou ce dont ils ont besoin. (Fournisseur 04)

Une relation fondée sur la responsabilité a permis de créer un climat de confiance, ce qui a permis d'accéder aux renseignements sur la santé

Elle est très... quand elle dit qu'elle va faire quelque chose, elle le fait. [...] Si elle dit, "Je serai là», elle y sera. (Participant 17)

Même lorsqu'elle n'était pas certaine d'une réponse à ma question, elle me disait : "Tu sais quoi, je vais me renseigner, je vais demander à [nom], je vais faire des recherches et je te reviendrai». (Participant 01)

Oui, et je me sens à l'aise de lui parler de ma douleur et de ce qui me gêne aujourd'hui, ou d'autres sujets. Vous savez, je me sens à l'aise làdedans, oui. Je pense que tout est une question de confiance pour moi, et si je ne fais pas confiance en quelqu'un et que j'ai l'impression que la personne n'essaie pas vraiment de m'aider, vous savez, je pourrais... je prends un peu de recul. Par contre, quand je sais qu'une personne me tend la main, je peux lui faire confiance. (Participant 06)

Si j'avais des questions sur... par exemple... elle m'expliquait, quand j'ai commencé à prendre le méthotrexate, si j'avais besoin de quelque chose, elle m’expliquait à quoi servait le médicament... et elle... me suggérait des choses. Par exemple, tu sais, peut-être demeurer assis un moment avant d'essayer de se lever. Des conseils utiles. (Participant 23)

Je pense que beaucoup d'entre eux ont commencé à comprendre ce qu'ils 
vivaient. Ils ont remarqué les moments où ils avaient, par exemple, des chiffres plus bas sur l'EQ5 [EQ-5D, une mesure de la qualité de vie]. Ils pouvaient savoir quand ils avaient une poussée, et ils aimaient suivre les tendances. (Agente de liaison avec les personnes atteintes d'arthrite)

\section{Facilitation des communications avec les fournisseurs de soins de santé}

Si le médecin a besoin d'informations et que je ne peux pas expliquer les mots correctement, [l'agente de liaison avec les personnes atteintes d'arthrite] peut le faire pour moi... (Participant 06)

Par exemple, la dernière fois que j'ai vu la $D^{\text {re }}$ [nom], j'ai oublié certaines choses que je voulais lui demander, et [l'agente de liaison avec les personnes atteintes d'arthrite] qui était là a simplement dit : «Oh oui, vous deviez lui demander telle ou telle chose. » Comme je l'ai dit, elle est ma mémoire, vous voyez? (Participant 13)

Je crois que cela a ouvert la communication, alors... les gens défendent davantage leurs intérêts... et sont davantage disposés à nous dire ce qui se passe entre les visites. (Fournisseur 01)

\section{Inciter à l'utilisation des ressources disponibles et permettre l'activation des plans de traitement}

Une partie d'entre eux n'arrêtait pas de dire qu'ils avaient besoin de ces ressources, mais lorsqu'il s'agissait de les utiliser et qu'elles se trouvaient juste à portée de main, ils ne les utilisaient pas ... Mais je pense que c'est en partie une question de confiance... (Agente de liaison avec les personnes atteintes d'arthrite)

Je pense que cela a eu un effet bénéfique... Encore une fois, cela augmente l'efficacité de ce que je dois faire parce que je n'ai pas à attendre le retour d'un résultat de laboratoire... ou à remplir à nouveau la demande. C’est déjà fait. (Fournisseur 02)

\section{Obstacles à la faisabilité}

Bien que l'intervention ait été généralement bien accueillie, nous avons dû relever certains défis pour que les participants maintiennent leur engagement envers ce modèle de soins. Divers problèmes ont surgi en raison de conflits d'horaire, de limites dans les télécommunications (numéros de téléphone inexacts ou plus en service) et de crises personnelles, familiales ou au sein de la collectivité.

L'agente de liaison avec les personnes atteintes d'arthrite était disponible uniquement pendant ses heures de travail, ce qui a constitué un problème logistique car cet horaire ne correspondait pas toujours au moment préféré des participants pour interagir. Les difficultés liées au transport ont également constitué un obstacle lorsque l'agente de liaison avec les personnes atteintes d'arthrite ne pouvait pas se rendre au domicile des patients ou que ces derniers ne pouvaient pas se rendre à la clinique.

Nous avons abordé le projet de manière longitudinale, en recherchant un financement permanent dans le cadre du budget opérationnel et en confiant le poste à une personne résidant dans la collectivité afin de pouvoir la soutenir et de lui permettre de s'acquitter efficacement de cette fonction particulière. Malheureusement, des priorités concurrentes de financement sont apparues dans la collectivité et il n'a pas été possible de maintenir ce poste après la fin du financement de l'étude.

\section{Analyse}

Le modèle de soins fondé sur l'agente de liaison avec les personnes atteintes d'arthrite, élaboré conjointement avec une collectivité autochtone, a aidé des patients autochtones souffrant d'arthrite à s'orienter parmi les services de santé pour répondre à leurs besoins et se concentrer sur leurs plans de soins personnels axés sur le patient. Tant les patients qui ont participé à notre étude que les fournisseurs de soins de santé de la collectivité ont été satisfaits de ce modèle de soins. Un patient a bien illustré la perception globale du modèle fondé sur l'agente de liaison avec les personnes atteintes d'arthrite lorsqu'il a dit que l'agente «ne fait pas de miracles qui fera disparaître mon arthrite, [mais] qu'elle est là pour vous aider sur le plan médical et pour vous apporter un soutien moral.»

L'orientation au sein du système de santé est complexe en matière de prise en charge des maladies chroniques. L'agente de liaison avec les personnes atteintes d'arthrite a fait le pont entre les fournisseurs de soins de santé et les patients et a favorisé la continuité des soins. Selon un métarésumé d'études qualitatives sur l'expérience des patients en matière de continuité des soins, la présence d'un seul clinicien de confiance qui aide le patient à prendre des décisions et à s'orienter dans le système est au cœur de ce que les gens perçoivent comme une continuité adéquate des soins ${ }^{26}$. Le soutien aux patients, la sensibilisation, la gestion des cas et l'orientation dans le système, assurés par un personnel formé envers lequel la collectivité a confiance, sont des approches efficaces dans la prise en charge des maladies chroniques, en particulier dans les collectivités vulnérables et mal desservies $^{27}$. Dans notre étude, le modèle de soins mis en place semble avoir permis aux patients de recevoir des soins coordonnés et continus au sein de la collectivité grâce au travail de l'agente de liaison avec les personnes atteintes d'arthrite.

Dans cette étude, menée dans des conditions réelles, ce ne sont pas tous les patients qui ont souhaité remplir les évaluations, et nous ne sommes en mesure de rendre compte que des perspectives et des résultats des patients qui ont pleinement participé au modèle de soins.

Nos conclusions sont probablement valables pour de nombreuses populations et collectivités autochtones du Canada.

\section{Remerciements}

Nous remercions Cheryl Sorenson et Margaret Kargard, des Siksika Health Services. Elles ont joué un rôle déterminant dans la mise en œuvre du modèle de soins.

Nous tenons également à remercier les membres de l'Arthritis Community Advisory Council, notamment Sharon Calf Robe, Tangee Scalplock, Lesley Stimson et Rosalyn Manyguns.

\section{Financement}

Cette étude a été financée par la Société de l'arthrite (SOG-14-130), l'Initiative canadienne pour des résultats en soins rhumatologiques et les Instituts de recherche en santé du Canada (subventions d'équipe dans le cadre de l'Initiative phare sur l'inflammation : PRECISION [Prévenir les complications des maladies inflammatoires de la peau, des articulations et de l'intestin, THC-135235, IRSC-IRSC : 0492004733 , FRN n $\left.\left.{ }^{\circ} 135235\right]\right)$. 
VU est financée par une bourse postdoctorale Eyes High de l'Université de Calgary. ALS a été financé par une bourse postdoctorale Banting des IRSC.

\section{Conflit d'intérêts}

Les auteurs déclarent qu'ils n'ont aucun conflit d'intérêts.

\section{Contributions des auteurs et avis}

VU : Analyse, rédaction de la première version du manuscrit puis examen et révision

ALS : Conceptualisation, analyse, acquisition de financement, enquêtes, méthodologie, administration du projet, supervision, rédaction de la première version du manuscrit puis examen et révision

VBC : Enquêtes, ressources, examen et révision du manuscrit

AR : Analyse, rédaction de la première version du manuscrit puis examen et révision

LoC : Conceptualisation, acquisition de financement, supervision, examen et révision du manuscrit

TK : Organisation de contenu, enquêtes, administration du projet, supervision, analyse, graphisme, examen et révision du manuscrit

LyC : Conceptualisation, acquisition de financement, méthodologie, supervision, examen et révision du manuscrit

TW : Conceptualisation, acquisition de financement, administration du projet, ressources, examen et révision du manuscrit

DL : Conceptualisation, acquisition de financement, enquêtes, méthodologie, administration du projet, supervision, examen et révision du manuscrit

CB : Conceptualisation, analyse, acquisition de financement, enquêtes, méthodologie, administration du projet, ressources, supervision, visualisation, rédaction de la première version du manuscrit puis examen et révision

Le contenu de l'article et les points de vue qui y sont exprimés n'engagent que les auteurs et ne correspondent pas nécessairement à ceux du gouvernement du Canada.

\section{Références}

1. Barnabe C, Hemmelgarn B, Jones CA, et al. Imbalance of prevalence and specialty care for osteoarthritis for first nations people in Alberta, Canada. J Rheumatol. 2015;42(2):323-328. https://doi.org/10.3899/jrheum.140551

2. Barnabe C, Jones CA, Bernatsky S, et al. Inflammatory arthritis prevalence and health services use in the First Nations and non-First Nations populations of Alberta, Canada. Arthritis Care Res (Hoboken). 2017;69(4):467474. https://doi.org/10.1002/acr.22959

3. Nagaraj S, Barnabe C, Schieir O, et al. Early rheumatoid arthritis presentation, treatment, and outcomes in Aboriginal patients in Canada: A Canadian Early Arthritis Cohort study analysis. Arthritis Care Res (Hoboken). 2018;70(8):12451250. https://doi.org/10.1002/acr.23470

4. Hurd K, Barnabe C. Systematic review of rheumatic disease phenotypes and outcomes in the Indigenous populations of Canada, the USA, Australia and New Zealand. Rheumatol Int. 2017;37(4):503-521. https://doi.org/10 $.1007 / \mathrm{s} 00296-016-3623-\mathrm{z}$

5. First Nations Information Governance Centre. National Report of the First Nations Regional Health Survey: Phase 3: Volume One. Ottawa (ON): FNIGC; 2018 Jul.

6. Reading CL, Wien F. Inégalités en matière de santé et déterminants sociaux de la santé des peuples autochtones. Prince (C.-B.) : Centre de collaboration nationale de la santé autochtone; 2009.

7. Agence de la santé publique du Canada. État de santé des Canadiens 2016 : Rapport de l'administrateur en chef de la santé publique. Ottawa (Ont.) : ASPC; 2016.

8. Allan B, Smylie J. First Peoples, second class treatment: the role of racism in the health and well-being of Indigenous peoples in Canada. Toronto (ON): Wellesley Institute; 2015.

9. Organisation mondiale de la Santé. Documents fondamentaux : quaranteneuvième édition (comprenant les amendements adoptés jusqu'au 31 mai 2019). Genève (Suisse) : Organisation mondiale de la Santé; 2020.
10. Thurston WE, Coupal S, Jones CA, et al. Discordant indigenous and provider frames explain challenges in improving access to arthritis care: a qualitative study using constructivist grounded theory. Int J Equity Health. 2014;13(1): 46. https://doi.org/10.1186/1475-9276 $-13-46$

11. Le portail canadien des pratiques exemplaires. Méthodes autochtones éprouvées. Bienvenue aux Méthodes autochtones éprouvées : Bonnes pratiques en santé publique dans les communautés autochtones [Internet]. Ottawa (Ont.) : Agence de la santé publique du Canada; 2015.

12. Commission de vérité et réconciliation Canada. Commission de vérité et réconciliation Canada : appels à l'action. Winnipeg (Man.) : Commission de vérité et réconciliation Canada; 2015.

13. Alkema GE, Wilber KH, Shannon GR, Allen D. Reduced mortality: the unexpected impact of a telephone-based care management intervention for older adults in managed care. Health Serv Res. 2007;42(4):1632-1650. https:// doi.org/10.1111/j.1475-6773.2006 .00668.x

14. Mitton C, O'Neil D, Simpson L, Hoppins Y, Harcus S. Nurse-Physician Collaborative Partnership: a rural model for the chronically ill. Can J Rural Med. 2007;12(4):208-216.

15. McBrien KA, Ivers N, Barnieh L, et al. Patient navigators for people with chronic disease: a systematic review. PLoS One. 2018;13(2):e0191980. https:// doi.org/10.1371/journal.pone.0191980

16. Askew DA, Togni SJ, Schulter PJ, et al. Investigating the feasibility, acceptability and appropriateness of outreach case management in an urban Aboriginal and Torres Strait Islander primary health care service: a mixed methods exploratory study. BMC Health Serv Res. 2016;16:178. https://doi.org /10.1186/s12913-016-1428-0

17. Feldman $\mathrm{CH}$, Wohlfahrt A, Campos A, et al. Can patient navigators improve adherence to disease-modifying antirheumatic drugs? Quantitative findings from a six-month single-arm pilot intervention. Arthritis Care Res (Hoboken). 2018;70(9):1400-1405. https://doi.org /10.1002/acr.23302 
18. Wohlfahrt A, Campos A, Iversen MD, et al. Use of rheumatology-specific patient navigators to understand and reduce barriers to medication adherence: Analysis of qualitative findings. PLoS One. 2018;13(7):e0200886. https:// doi.org/10.1371/journal.pone.0200886

19. Lu MC, Guo HR, Livneh H, Lin MC, Lai NS, Tsai TY. The effectiveness of nurse-led case management for patients with rheumatoid arthritis in Taiwan. Int J Clin Pract. 2020;74(2):e13443. https://doi.org/10.1111/ijcp.13443

20. Nagaraj S, Kargard M, Hemmelgarn $B$, Fritzler M, White T, Barnabe C. Effectiveness of an outreach model of care for rheumatology specialty clinics to an on-reserve First Nations community. Int J Indig Health. 2018; 13(1):156-166. https://doi.org/10.32799 /ijih.v13i1.30315

21. Barber CE, Thorne JC, Ahluwalia V, et al. Feasibility of measurement and adherence to system performance measures for rheumatoid arthritis in 5 models of care. J Rheumatol. 2018; 45(11):1501-1508. https://doi.org/10 $.3899 /$ jrheum. 171284

22. Loyola-Sanchez A, Pelaez-Ballestas I, Crowshoe L, et al. "There are still a lot of things that I need" : a qualitative study exploring opportunities to improve the health services of First Nations People with arthritis seen at an on-reserve outreach rheumatology clinic. BMC Health Serv Res. 2020; 20(1):1076. https://doi.org/10.1186 /s12913-020-05909-9

23. Chamberlain R, Rapp CA. A decade of case management: a methodological review of outcome research. Community Ment Health J. 1991;27(3): 171-188. https://doi.org/10.1007 /BF00752419

24. Brown AJ, Varcoe C, Smye V, ReimerKirkham S, Lynam MJ, Wong S. Cultural safety and the challenges of translating critically-oriented knowledge in practice. Nurs Philos An Int J Health Care Prof. 2009;10:167-179. https://doi.org/10.1111/j.1466-769X .2009.00406.x

25. Centre de gouvernance de l'information des Premières Nations. Les principes de $\mathrm{PCAP}^{\circledR}$ des Premières Nations [Internet]. Akwesasne (Ont.) : CGIPN; [consultation le 1er déc. 2020]. En ligne à : https://fnigc.ca/fr/les-principes -de-pcap-des-premieres-nations/
26. Haggerty JL, Roberge D, Freeman GK, Beaulieu C. Experienced continuity of care when patients see multiple clinicians: a qualitative metasummary. Ann Fam Med. 2013;11(3):262-271. https:// doi.org/10.1370/afm.1499

27. Kim K, Choi JS, Choi E, et al. Effects of community-based health worker interventions to improve chronic disease management and care among vulnerable populations: a systematic review. Am J Public Health. 2016; 106(4):e3-e28. https://doi.org/10.2105 /AJPH.2015.302987 